\title{
Small interfering RNA-directed targeting of RON alters invasive and oncogenic phenotypes of human hepatocellular carcinoma cells
}

\author{
SUNG-BUM CHO $^{1 *}$, YOUNG-LAN PARK ${ }^{1 *}$, YOUNG-A SONG ${ }^{1}$, KYU-YEOL KIM ${ }^{1}$, GI-HOON LEE ${ }^{1}$, \\ DAE-HO CHO ${ }^{1}$, DAE-SEONG MYUNG ${ }^{1}$, KANG-JIN PARK ${ }^{1}$, WAN-SIK LEE ${ }^{1}$, IK-JOO CHUNG ${ }^{1}$, \\ SUNG-KYU CHOI ${ }^{1}$, KYUNG-KEUN KIM $^{2}$ and YOUNG-EUN JOO ${ }^{1}$
}

Departments of ${ }^{1}$ Internal Medicine and ${ }^{2}$ Pharmacology, Chonnam National

University Medical School, Gwangju, Republic of Korea

Received June 3, 2011; Accepted July 26, 2011

DOI: $10.3892 /$ or.2011.1435

\begin{abstract}
The recepteur d'origine nantais (RON) receptor tyrosine kinase is highly expressed in various cancers including human hepatocellular carcinoma (HCC) and involved in tumor progression. The aims of the current study were to evaluate whether RON affects tumor cell behavior and oncogenic signaling cascades in HCC cells. We investigated the biologic role of RON on tumor cell behavior and oncogenic signaling cascades including Akt, c-Raf and extracellular signal-regulated kinase (ERK) by using the small interfering RNA (siRNA) in HCC cell lines, chang, HepG2 and Huh7. Knockdown of RON suppressed tumor cell migration and invasion in all tested HCC cell lines. The proportion of apoptotic cells induced by knockdown of RON was greater than that induced by transfection of the scramble siRNA in all tested HCC cell lines. Knockdown of RON resulted in cell cycle arrest in the $\mathrm{G}_{2} / \mathrm{M}$ phase of chang and Huh7 cells, and sub $\mathrm{G}_{1}$ phase of HepG2 cells. Knockdown of RON activated cleaved caspase-3 and PARP, and down-regulated the expression of $\mathrm{Bcl}-2, \mathrm{Bcl}-\mathrm{xL}$ and survivin, leading to induction of apoptosis in all tested cell lines. Knockdown of RON negatively regulates the progression of the cell cycle by decreasing cyclin D1 and D3, and increasing p21 and p27 in all tested cell lines. The phosphorylation of Akt, c-Raf and ERK1/2 signal proteins was significantly blocked by knockdown of RON in all tested cell lines. These results suggest that RON is associated with invasive and oncogenic phenotypes such as tumor cell migration, invasion, resistance to apoptosis and cell cycle arrest through
\end{abstract}

Correspondence to: Dr Young-Eun Joo, Department of Internal Medicine, Chonnam National University Medical School, 8 Hak-Dong, Dong-ku, Gwangju 501-757, Republic of Korea

E-mail: yejoo@chonnam.ac.kr

${ }^{*}$ Contributed equally

Key words: recepteur d'origine nantais, hepatocellular carcinoma, tumor progression, signal transduction the modulation of Akt, c-Raf and ERK signaling cascades in HCC cells.

\section{Introduction}

Human hepatocellular carcinoma (HCC) is the fifth most common cancer worldwide and the third most common cause of death from cancer. Early diagnosis of HCC is rare, and most persons present with locally advanced or metastatic disease. Accordingly, only 10-30\% of newly diagnosed HCC persons are eligible for radical treatment such as resection, liver transplantation, and percutaneous ablation techniques (1-3). Thus the inhibition of metastasis is of great importance in the therapy of HCC. Cancer metastasis is a complex process and arises through multiple genetic alterations including mutational activation of oncogenes and inactivation of tumor suppressor genes $(4,5)$.

Among the oncogenes activated in the variety of cancers, receptor tyrosine kinases (RTKs) play a critical role in regulating cell growth, differentiation and survival, and are also directly linked to cancer development and metastasis (6-12). The recepteur d'origine nantais $(\mathrm{RON})$ has features of a classical receptor tyrosine kinase and belongs to the MET proto-oncogene family. RON is a heterodimeric protein of disulfide-linked extracellular $\alpha$ and transmembrane $\beta$ subunits with intrinsic tyrosine kinase activity derived from proteolytic cleavage of a single-chain precursor of $170 \mathrm{kDa}$ (13-19). Transgenic mouse models overexpressing RON in lung or mammary epithelial cells develop lung and breast carcinomas and are associated with metastatic dissemination $(20,21)$. RON is overexpressed in a variety of human epithelial cancers including breast, colon, lung, ovary, pancreas and bladder (22-27). Moreover, RON overexpression is associated with poor survival as well as metastasis in breast, colon and bladder cancers $(22,23,27)$. Also, RON has been shown to be overexpressed at mRNA and protein levels in HCC (28). Various cytokines including hepatocyte growth factor, TNF- $\alpha$, IL-1 and IL-6 increase RON expression in lipopolysaccharideinduced murine model of acute liver failure and HCC cell line (28-30). Therefore, induction of RON by these cytokines may 
play an important role in development and progression of HCC. However, the impact of RON on HCC progression has not yet to be fully established.

Activation of the RON is essential in transducing a variety of intracellular signaling cascades including the phosphatidylinositol-3 kinase (PI3K)/Akt, mitogen-activated protein kinase (MAPK) including extracellular signal-regulated kinase (ERK), $\beta$-catenin and nuclear factor- $\kappa \mathrm{B}(\mathrm{NF}-\kappa \mathrm{B})$ that lead to cellular growth, motility and invasion of extracellular matrices (13-19).

Akt causes a variety of biological effects, including cell survival and proliferation, whereas c-Raf is a serine/threonine kinase, implicated in the induction of growth arrest and differentiation. c-Raf is one of the downstream targets for Akt and phosphorylated and inactivated by Akt activation. c-Raf phosphorylates and activates ERK signaling $(31,32)$. However, the impact of RON on Akt, c-Raf and ERK signaling cascades in HCC cells remains unknown.

The aims of the current study were to evaluate whether RON affects tumor cell behavior and oncogenic signaling cascades including Akt, c-Raf and ERK signaling in HCC cells.

\section{Materials and methods}

Cell culture and small interfering RNA (siRNA) transfection. HCC cell lines, chang, HepG2 and Huh7 were obtained from the American Type Culture Collection line Inc. and cultured in DMEM (Hyclone, Loan, UT, USA) supplemented with $10 \%$ fetal bovine serum (Hyclone), $50 \mathrm{U} / \mathrm{ml}$ penicillin, and $50 \mu \mathrm{g} / \mathrm{ml}$ streptomycin (Gibco, NY, USA). To transfect siRNA, HCC cells were seeded into a plate such that they were $40-50 \%$ confluent at the time of transfection. RON siRNA (Santa Cruz Biotechnology, Santa Cruz, CA, USA) and scramble siRNA (Qiagen, MD, USA) were transfected with lipofectamine ${ }^{\mathrm{TM}}$ RNAiMAX (Invitrogen, Carlsbad, CA, USA) according to the manufacturer's recommendations and then incubated up to $48 \mathrm{~h}$.

Cell migration assay. Cells were cultured in a 6-well plate and were transfected with $100 \mathrm{pM}$ of RON and scramble siRNA. After incubation for $48 \mathrm{~h}$, scratch wound was carried out by creating a linear cell-free region using $200 \mu$ l pipette tip resulting in a uniform gap. The media were changed to $5 \%$ FBS/RPMI-1640 media. The progress of cell migration into the scratched area was photographed at $0,6,12$ and $24 \mathrm{~h}$ using an inverted microscope. The distances between gaps were normalized to $1 \mathrm{~cm}$ after capture of six random sites.

Cell invasion assay. Transwell filters (8.0 $\mu \mathrm{m}$ pores) were coated with $1 \%$ gelatin/RPMI-1640 overnight and dried out at room temperature. Cells transfected with siRNA were seeded at $2 \times 10^{5}$ cells in $120 \mu 10.2 \%$ bovine serum albumin (BSA) medium in the upper chamber. Subsequently, $400 \mu 10.2 \%$ BSA medium containing $20 \mu \mathrm{g} / \mathrm{ml}$ human plasma fibronectin (Calbiochem, La Jolla, CA, USA), a chemotactic factor, were loaded into the lower chamber. After incubation for $24 \mathrm{~h}$, migrated cells on bottom surface of the transwell were stained with Diff Quik solution (Sysmex, Kobe, Japan) and counted in 5 selected fields under a light microscope. Data were expressed as the mean \pm standard error (SE) of the number of cells/field in three individual experiments.
Apoptosis analysis. The collected RON knockdown HCC cells using trypsin were washed with PBS and resuspended in 1X binding buffer (BD Biosciences, San Diego, CA, USA). Cell suspensions were incubated in APC Annexin V and 7-aminoactinomycin D (7-AAD) (BD Biosciences) for $20 \mathrm{~min}$ at RT. Prior to flow cytometric analysis $400 \mu \mathrm{l}$ of $1 \mathrm{X}$ binding buffer was added and cells were analyzed on a FACSCalibur flow cytometer (Becton-Dickinson, San Jose, CA, USA) using CellQuest software.

Cell cycle analysis. The RON and scramble siRNA transfected $\mathrm{HCC}$ cells were harvested using trypsin. Cell pellets were washed with cold phosphate-buffered saline (PBS) and were fixed in ice-cold $70 \%$ ethanol to determine cell cycle distribution. The cells fixed in $70 \%$ ethanol were washed with PBS and resuspended in $0.1 \%$ sodium citrate, $0.1 \%$ Triton $\mathrm{X}-100$ and $50 \mu \mathrm{g} / \mathrm{ml}$ propidium iodide (PI) for $20 \mathrm{~min}$ at room temperature in the presence of $10 \mu \mathrm{g} / \mathrm{ml}$ ribonuclease A (propidium iodide and ribonuclease A were from Sigma-Aldrich). At least 10,000 events were collected in each histogram. Cell cycle analysis was performed using the BD CellQuest ${ }^{\circledR}$ version 3.3 (Becton-Dickinson) and WinMDI version 2.9 (The Scripps Research Institute, San Diego, CA, USA).

RNA isolation and amplification by RT-PCR. RNA was isolated using the TRIzol method (Invitrogen), reverse transcribed, and amplified as previously described using specific primers for RON and GAPDH. Primer sequences were as follows; RON, 5'-TCG CCT CGA TGG AGC TCC TC-3'/5'-CAT GTG TGC CAC TGT GAC GT-3'; GAPDH, 5'-ACC ACA GTC CAT GCC ATC AC-3'/5'-TCC ACC ACC CTG TTG CTG TA-3'. For each sample, $1 \mu \mathrm{g}$ total-RNA was used to prepare cDNA in a reaction containing $50 \mathrm{ng} / \mu \mathrm{l}$ oligo(dt) (Promega, Madison, WI, USA) and after incubation at $72^{\circ} \mathrm{C}$ for $10 \mathrm{~min}, \mathrm{MMLV}$ reverse transcriptase (Invitrogen) and RNAsin (Takara, Otsu, Shiga, Japan) were added and incubation continued at $42^{\circ} \mathrm{C}$ for $1 \mathrm{~h}$ and at $72^{\circ} \mathrm{C}$ for $15 \mathrm{~min}$. PCR amplification of cDNA was performed using gene-specific primers and GoTaq. Polymerase (Promega). PCR products were separated by electrophoresis on $1 \%$ agarose gel containing ethidium bromide.

Western blotting. Total cell extracts were lysed in M-PER ${ }^{\circledR}$ mammalian protein extraction reagent (Thermo, Rockford, IL, USA) with Halt ${ }^{\mathrm{TM}}$ phosphatase inhibitor and Halt ${ }^{\mathrm{TM}}$ protease inhibitor cocktail (Thermo). Protein concentrations were determined by $\mathrm{BCA}^{\mathrm{TM}}$ protein assay (Thermo). The proteins were separated by 10 or $12 \%$ SDS-PAGE and transferred onto PVDF membrane (Millipore, Billerica, MA, USA). The following antibodies were used; antibodies against RON ( $\beta$-chain), survivin and GAPDH were purchased from Santa Cruz Biotechnology. Antibodies against phospho-Akt (Ser473), phospho-Akt (Thr308), Akt, phospho-c-Raf (Ser259), cleaved caspase-3, cleaved Poly (ADP-ribose) polymerase (PARP), cyclin dependent kinase 4 (CDK4), cyclin D1, cyclin D3, p21, p27, Bad, Bid, $\mathrm{Bcl}-2$ and $\mathrm{Bcl}-\mathrm{xL}$ were purchased from Cell Signaling (Danvers, MA, USA). Immunoreactive proteins were visualized by the enhanced chemiluminescence detection system HRP substrate (Millipore). Immunoreactive bands were quantified using the luminescent image analyzer LAS-4000 and MultiGauge V3.2 image analyzer software (Fujifilm, Tokyo, Japan). 
A
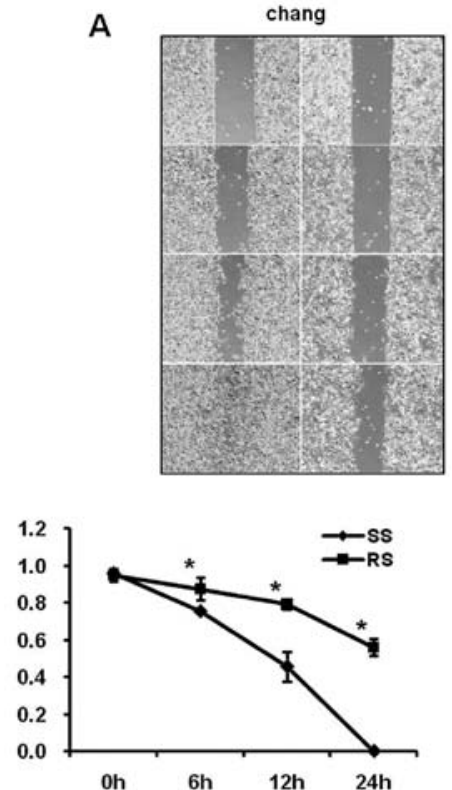
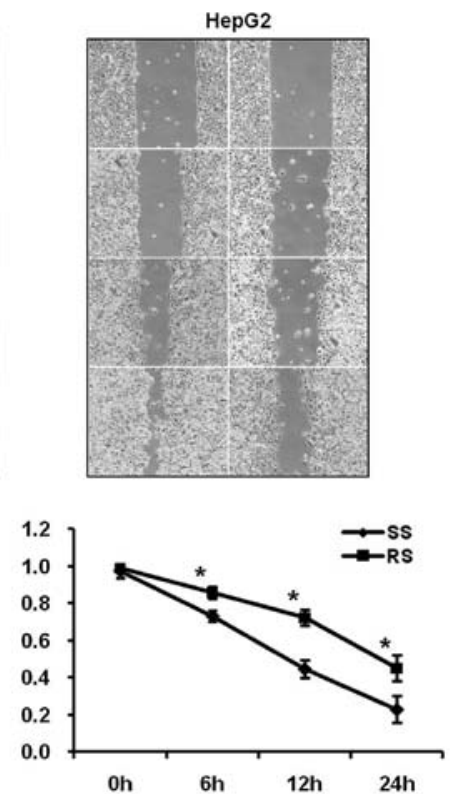
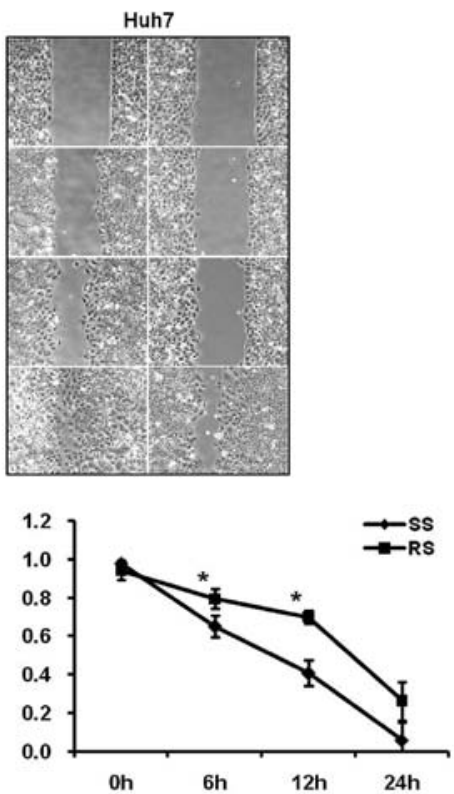
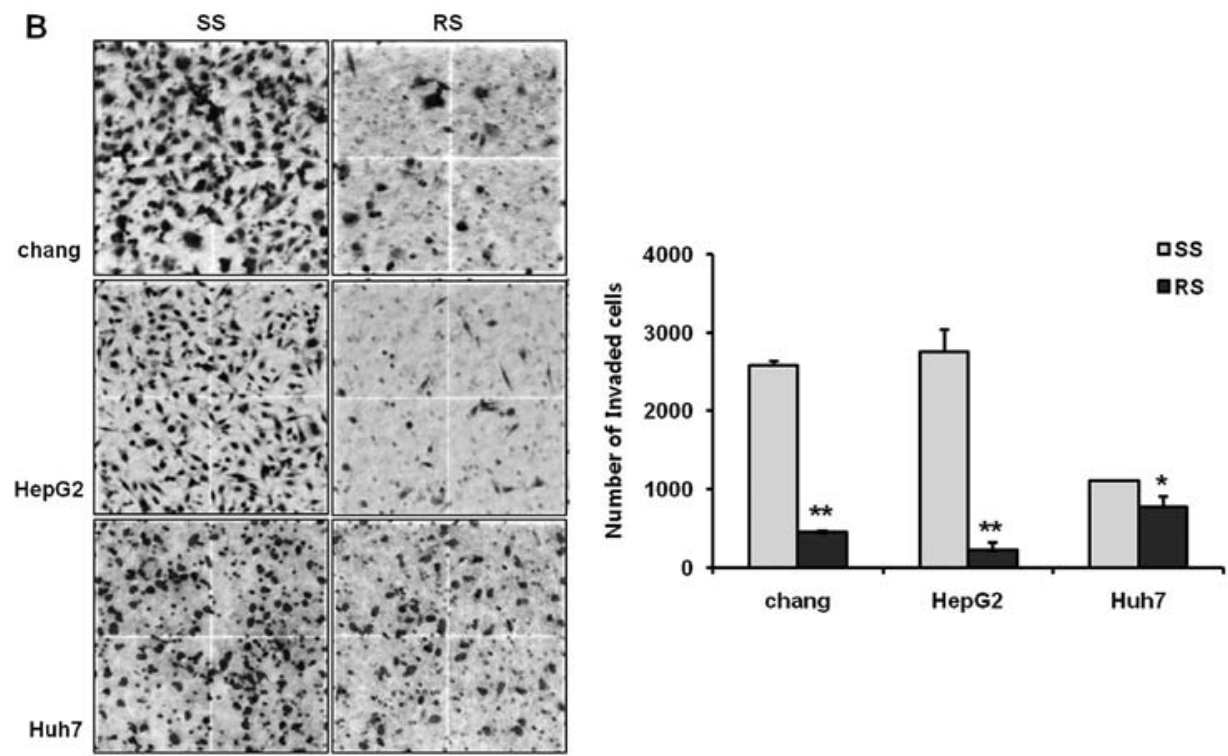

Figure 1. Knockdown of RON suppresses tumor cell migration and invasion of HCC cells. (A) Effect of RON knockdown on cell migration. The wound healing assay using the siRNA-transfected cells was performed and graphs of cell migration are displayed as relative healing distances (mean $\pm \mathrm{SE}, \mathrm{n}=3$; $\left.^{*} \mathrm{P}<0.05\right)$. The artificial wound gap in plates of scramble siRNA-transfected chang, HepG2 and Huh7 cells became significantly narrower than that in RON siRNA-transfected cells at 6, 12 and $24 \mathrm{~h}\left({ }^{*} \mathrm{P}<0.05\right)$. (B) Invasion assay of cells under RON knockdown. The number of invading RON siRNA-transfected chang, HepG2 and Huh7 cells were significantly lower than that of scramble siRNA-transfected cells (mean $\pm \mathrm{SE}, \mathrm{n}=6$; ${ }^{* *} \mathrm{P}<0.005,{ }^{*} \mathrm{P}<0.05$ ). SS, scramble siRNA; RS, RON siRNA.

Statistical analysis. To compare intergroups, data were derived from at least three independent experiments. The data are presented as the means \pm SD. In intergroup comparisons, a student's t-test was used to determine statistical significance. The Statistical Package for the Social Sciences used was (SPSS/ $\mathrm{PC}+15.0$, Chicago, IL). P-values $<0.05$ were considered to indicate statistical significance.

\section{Results}

Knockdown of RON suppresses tumor cell migration and invasion of HCC cells. To evaluate the impact of RON knockdown on migration and invasion of HCC cells, chang, HepG2 and
Huh7 cells, the migration and invasion assays using siRNAtransfected cells were performed. RON gene expression in all tested cells showed a specific reduction at mRNA and protein levels by transfection of RON siRNA (data not shown). The artificial wound gap in plates of scramble siRNA-transfected chang, HepG2 and Huh7 cells became significantly narrower than that in RON siRNA-transfected cells at 6, 12 and $24 \mathrm{~h}\left({ }^{*} \mathrm{P}<0.05\right)$ (Fig. 1A). The number of invading RON siRNA-transfected chang, HepG2 and Huh7 cells were 449.3 $\pm 25.4,223.7 \pm 100.3$ and $780.0 \pm 133.0$, whereas for the scramble siRNA-transfected cells they were $2577.3 \pm 67.0,2752.0 \pm 290.0$ and $1102.7 \pm 19.6$ as measured by the five random squares of the $0.5 \times 0.5 \mathrm{~mm}^{2}$ microscope fields under the conditions of $10 \mu \mathrm{g} / \mathrm{ml}$ fibronectin; 
A
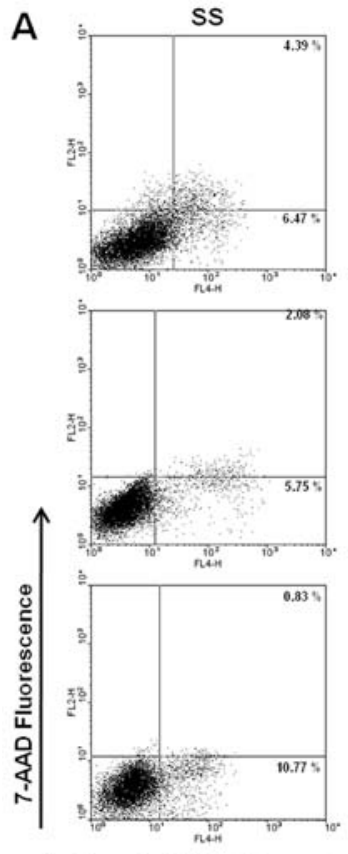

Annexin V-APC Fluorescence
B

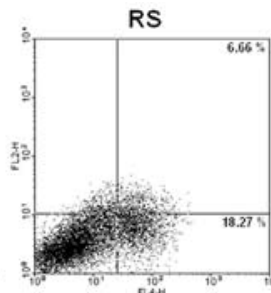

chang
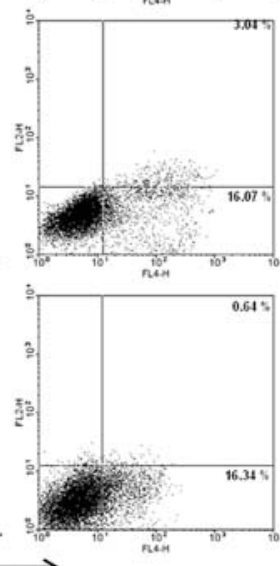

HepG2

Huh7
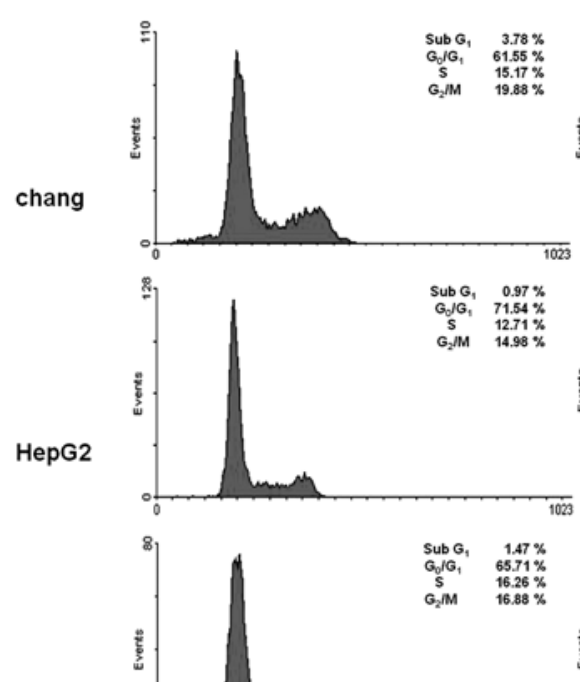

Huh7

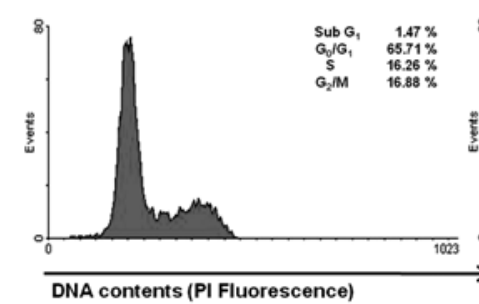

RS
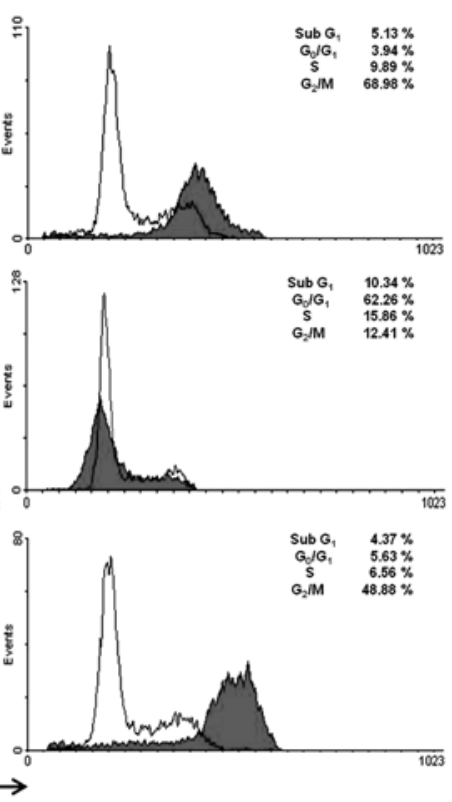

Figure 2. Knockdown of RON induces apoptosis and cell cycle arrest in HCC cells. (A) The proportion of early apoptotic cells induced by transfection of RON siRNA was greater than that induced by transfection of the scramble siRNA (18.27 vs. 6.47 , 16.07 vs. 5.75, and 16.34 vs. $10.77 \%$, respectively) in chang, HepG2 and Huh7 cells. (B) Knockdown of RON induced cell cycle arrest of the $\mathrm{G}_{2} / \mathrm{M}$ phase in chang and Huh7 cells and $\mathrm{G}_{0} / \mathrm{G}_{1}$ phase in HepG2 cells. One representative experiment of the three independent experiments is shown. SS, scramble siRNA; RS, RON siRNA.

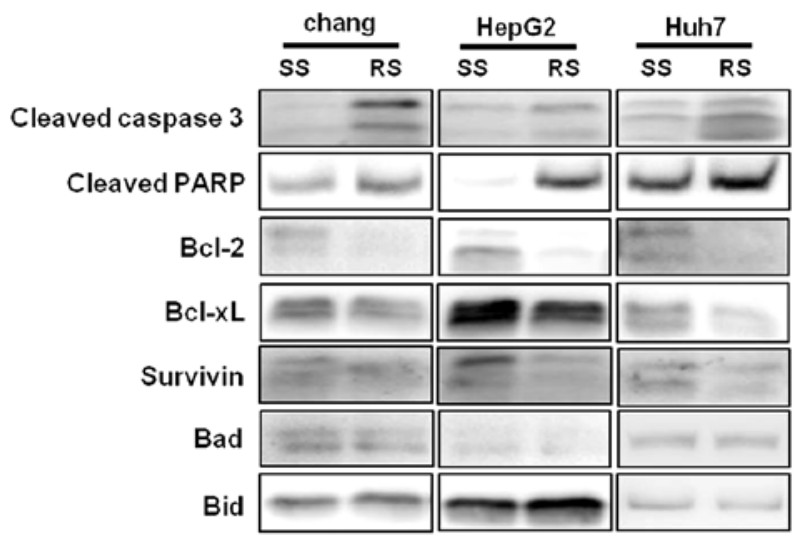

Figure 3. RON knockdown-induced apoptosis is associated with the modulation of apoptotic regulatory proteins in HCC cells. (A) An increase in cleaved caspase-3 and PARP expression was detected in the chang, HepG2 and Huh7 cells after knockdown of RON. (B) RON knockdown led to a decrease of antiapoptotic proteins, Bcl-2, Bcl-xL and survivin in chang, HepG2 and Huh7 cells. PARP, Poly (ADP-ribose) polymerase; SS, scramble siRNA; RS, RON siRNA.

the difference between the two was statistically significant in chang, HepG2 and Huh7 cells $(\mathrm{P}<0.005, \mathrm{P}<0.005$ and $\mathrm{P}<0.05$, respectively) (Fig. 1B).

Knockdown of RON induces apoptosis and cell cycle arrest in HCC cells. The imbalance between apoptosis and cell cycle progression is closely related to cancer development and progression. To evaluate the impact of RON on apoptosis and cell cycle, we performed FACS analyses. The proportion of early apoptotic cells induced by transfection of RON siRNA was greater than that induced by transfection of the scramble
siRNA (18.27 vs. $6.47,16.07$ vs. 5.75 and 16.34 vs. $10.77 \%$, respectively) in chang, HepG2 and Huh7 cells (Fig. 2A). Knockdown of RON-induced cell cycle arrest of the $\mathrm{G}_{2} / \mathrm{M}$ phase in chang and Huh7 cells and sub $G_{1}$ phase in HepG2 cells (Fig. 2B). These results suggest that RON enhances growth and survival capacity in HCC cells.

RON knockdown-induced apoptosis is associated with the modulation of apoptotic regulatory proteins in HCC cells. Caspase-3 and PARP are the crucial mediators of apoptosis. An increase in cleaved caspase- 3 and PARP expression was detected in the chang, HepG2 and Huh7 cells after knockdown of RON. Apoptosis is regulated by numerous genes including pro-apoptotic and anti-apoptotic genes. RON knockdown led to a decrease of anti-apoptotic proteins, Bcl-2, Bcl-xL and survivin in chang, HepG2 and Huh7 cells. The pro-apoptotic protein levels such as Bad and Bid were not altered in response to RON knockdown in chang, HepG2 and Huh7 cells (Fig. 3).

Knockdown of RON induces cell cycle arrest through the modulation of cell cycle regulators in HCC cells. Cell cycle progression is positively regulated by a family of cyclins and CDKs and negatively by CDK inhibitors (CDKIs). The cyclin D1 and D3 level was significantly decreased by RON knockdown in all tested cells. The p21 and p27 protein level was significantly increased by RON knockdown in all tested cells. CDK4 protein level was not altered in response to RON knockdown (Fig. 4).

Knockdown of RON decreases the phosphorylation of Akt, c-Raf and ERK signaling proteins in HCC cells. We studied the effect of RON on Akt, c-Raf and ERK signaling pathways, essential for cell growth and survival during carcinogenesis. 


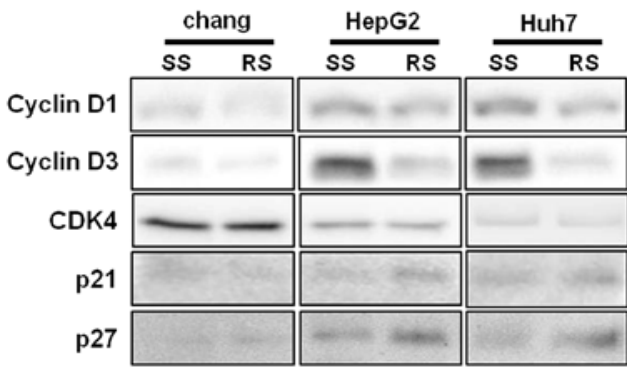

Figure 4. Knockdown of RON induces cell cycle arrest through the modulation of cell cycle regulators in HCC cells. The cyclin D1 and D3 levels were significantly decreased by RON knockdown in all tested cells. The p21 and p27 protein level was significantly increased by RON knockdown in all tested cells. CDK4 protein level was not altered in response to RON knockdown. SS, scramble siRNA; RS, RON siRNA.

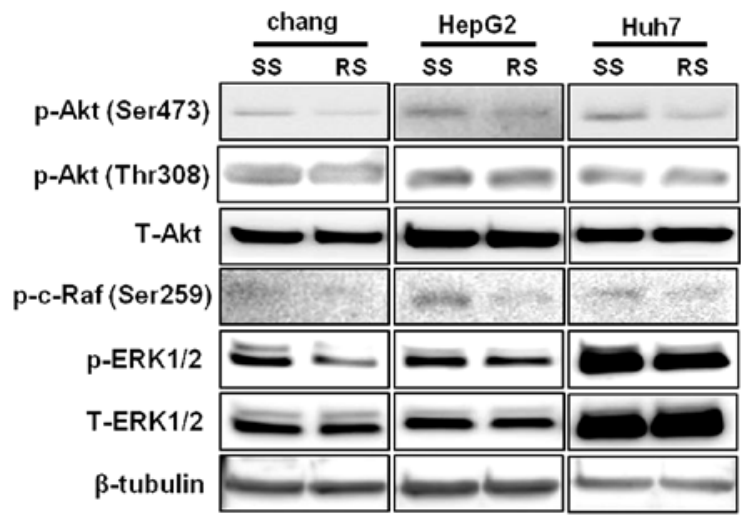

Figure 5. Knockdown of RON decreases the phosphorylation of Akt, c-Raf and ERK signaling proteins in HCC cells. The phosphorylation levels on Ser473 of Akt were decreased by RON knockdown in all tested cells. The phosphorylation levels on Thr308 of Akt were not altered by RON knockdown in all tested cells. The phosphorylation level of c-Raf was decreased by RON knockdown in all tested cells. The phosphorylation level of ERK1/2 was decreased by RON knockdown in all tested cells. SS, scramble siRNA; RS, RON siRNA.

The phosphorylation levels on Ser473 of Akt were decreased by RON knockdown in all tested cells. The phosphorylation levels on Thr308 of Akt were not altered by RON knockdown in all tested cells. The phosphorylation level of c-Raf (Ser259) was decreased by RON knockdown in all tested cells. The phosphorylation level of ERK1/2 was decreased by RON knockdown in all tested cells (Fig. 5).

\section{Discussion}

RON activates an array of oncogenic signaling cascades such as phosphoinositide-3-kinase/Akt, focal adhesion kinase, and MAPKs which are involved in cell proliferation, tubular morphogenesis, cell motility, migration and invasion of many cancer cells. RON modulates diverse processes involved in tumor progression and metastasis in a variety of human cancers (13-27). Consequently, an understanding of the molecular alterations of RON that facilitate tumor progression and metastasis will provide insight into approaches to optimized target therapies. However, the impact of RON on invasive and oncogenic phenotypes of HCC cells remains unknown.
We first studied the impact of RON knockdown on tumor cell migration and invasion, crucial to tumor metastasis in HCC cells. In our study, knockdown of RON suppressed tumor cell migration and invasion in tested HCC cells. These results suggest that modulation of RON could affect metastatic tumor progression in HCC.

Apoptosis and cell cycle plays an important role in supporting proper development and maintaining healthy homeostasis of mature tissues. The deregulation of apoptosis and cell cycle could be closely related to cancer development and progression (33-36). So, we next studied the effect of RON knockdown on apoptosis and cell cycle progression in HCC cells. Our results showed that RON knockdown induced apoptosis and cell cycle arrest in tested HCC cells. Apoptosis was induced by a decrease of anti-apoptotic proteins and cell cycle arrest induced by a decrease of cyclins and an increase of CDKIs. These results indicate that $\mathrm{RON}$ induces resistance to apoptosis and cell cycle arrest via the modulation of apoptotic and cell cycle regulatory proteins.

Amplification and/or overexpression of RON transduces oncogenic signals that regulate tumorigenic activities of a majority of cancer cell lines examined (13-19). Akt is a serine/ threonine protein kinase and involved in a wide variety of cellular functions including cellular proliferation, survival and differentiation. Activation of Akt has oncogenic effects and is reported to be up-regulated in various cancers. Akt is activated through the phosphorylation of two regulatory sites including Thr308 in the activation loop within the kinase domain and Ser473 in the C-terminal regulatory domain by upstream kinases such as phosphoinositide-dependent kinase 1 (37).

Next, we studied the effect of RON on Akt signaling pathway, essential for cell apoptosis and proliferation during carcinogenesis. In our study, the phosphorylation levels on Ser473 of Akt were decreased by RON knockdown in all tested cells. But the phosphorylation levels on Thr308 of Akt were not altered by RON knockdown in the tested cells. These results indicated that Akt signal was inactivated by phosphorylation of Ser473, but not Thr308 after knockdown of RON in HCC cells.

Activation of protein kinase, c-Raf mediates various cellular responses, such as proliferation, growth arrest, apoptosis and differentiation. Activation of Akt phosphorylates on Ser259 of c-Raf and results in a decrease in c-Raf activity $(31,32)$. In our study, the phosphorylation level of c-Raf (Ser259) was decreased by RON knockdown in all tested cells.

MAPK/ERK signaling is involved in many cellular programs such as cell growth, migration, survival and differentiation. Activation of RON leads to activation of its downstream target, MAPK/ERK signaling cascade (13-19). c-Raf phosphorylates and activates mitogen-activated protein kinase (MEK), and MEK phosphorylates ERK, leading to activation of $\operatorname{ERK}(38,39)$. In our study, the phosphorylation of ERK1/2 was decreased by RON knockdown in all tested cells. Therefore, knockdown of RON blocks Akt activation, and inhibits phosphorylation and inactivation of c-Raf and ERK1/2. To our knowledge, this is the first investigation on the effects of RON knockdown on the Akt, c-Raf and ERK signaling in HCC cells.

In summary, knockdown of RON suppressed tumor cell migration and invasion in HCC cells. Knockdown of RON induced apoptosis and cell cycle arrest in HCC cells. Knockdown 
of RON blocked the phosphorylation of Akt, c-Raf and ERK1/2 signal proteins in HCC cells. Taken together, RON is associated with invasive and oncogenic phenotypes such as tumor cell migration, invasion, resistance to apoptosis and cell cycle arrest through the modulation of Akt, c-Raf and ERK signaling cascades in HCC cells.

\section{Acknowledgements}

This study was supported by a grant (0720570) from the National R\&D Program for Cancer Control, Ministry of Health and Welfare, Republic of Korea, and partly by a research funds of Research Institute of Clinical Medicine, Chonnam National University Hospital in 2011 (CRI 11050-1), Republic of Korea.

\section{References}

1. Bruix J and Sherman M: Management of hepatocellular carcinoma. Hepatology 42: 1208-1236, 2005.

2. Song TJ, Ip EW and Fong Y: Hepatocellular carcinoma: current surgical management. Gastroenterology 127 (Suppl 1): S248-S260, 2004

3. Marrero JA, Fontana RJ, Barrat A, Askari F, Conjeevaram HS, Su GL and Lok AS: Prognosis of hepatocellular carcinoma: comparison of 7 staging systems in an American cohort. Hepatology 41: 707-716, 2005.

4. Chambers AF, Groom AC and MacDonald IC: Dissemination and growth of cancer cells in metastatic sites. Nat Rev Cancer 2 563-572, 2002

5. Townson JL and Chambers AF: Dormancy of solitary metastatic cells. Cell Cycle 5: 1744-1750, 2006.

6. Robertson SC, Tynan JA and Donoghue DJ: RTK mutations and human syndromes: when good receptors turn bad. Trends Genet 16: 265-271, 2000

7. Manning G, Whyte DB, Martinez R, Hunter T and Sudarsanam S The protein kinase complement of the human genome. Science 298: 1912-1934, 2002

8. Van de Woude GF, Jeffers M, Cortner J, Alvord G, Tsarfaty I and Resau J: Met-HGF/SF: tumorigenesis, invasion and metastasis. Ciba Found Symp 212: 119-130, 1997.

9. Yarden Y: Biology of HER2 and its importance in breast cancer. Oncology 61: 1-13, 2001.

10. Schlessinger J: Cell signaling by receptor tyrosine kinases. Cell 103: 211-225, 2000.

11. van der Geer P, Hunter T and Lindberg RA: Receptor proteintyrosine kinases and their signal transduction pathways. Annu Rev Cell Biol 10: 251-337, 1994.

12. Hanahan D and Weinberg RA: The hallmarks of cancer. Cell 100: 57-70, 2000.

13. Lu Y, Yao HP and Wang MH: Multiple variants of the RON receptor tyrosine kinase: biochemical properties, tumorigenic activities, and potential drug targets. Cancer Lett 257: 157-164, 2007.

14. Leonis MA, Thobe MN and Waltz SE: Ron-receptor tyrosine kinase in tumorigenesis and metastasis. Future Oncol 3: 441-448, 2007.

15. Camp ER, Liu W, Fan F, Yang A, Somcio R and Ellis LM: RON, a tyrosine kinase receptor involved in tumor progression and metastasis. Ann Surg Oncol 12: 273-281, 2005.

16. Wang MH, Yao HP and Zhou YQ: Oncogenesis of RON receptor tyrosine kinase: a molecular target for malignant epithelial cancers. Acta Pharmacol Sin 27: 641-650, 2006.

17. Wang MH, Wang D and Chen YQ: Oncogenic and invasive potentials of human macrophage-stimulating protein receptor, the RON receptor tyrosine kinase. Carcinogenesis 24: 1291-1300, 2003

18. Wagh PK, Peace BE and Waltz SE: Met-related receptor tyrosine kinase Ron in tumor growth and metastasis. Adv Cancer Res 100: $1-33,2008$.
19. Danilkovitch-Miagkova A: Oncogenic signaling pathways activated by RON receptor tyrosine kinase. Curr Cancer Drug Targets 3: 31-40, 2003.

20. Chen YQ, Zhou YQ, Fu LH, Wang D and Wang MH: Multiple pulmonary adenomas in the lung of transgenic mice overexpressing the RON receptor tyrosine kinase. Recepteur d'origine nantais. Carcinogenesis 23: 1811-1819, 2002.

21. Peace BE, Toney-Earley K, Collins MH and Waltz SE: Ron receptor signaling augments mammary tumor formation and metastasis in a murine model of breast cancer. Cancer Res 65: 1285-1293, 2005.

22. Maggiora P, Marchio S, Stella MC, et al: Overexpression of the RON gene in human breast carcinoma. Oncogene 16: 2927-2933, 1998.

23. Lee CT, Chow NH, Su PF, Lin SC, Lin PC and Lee JC: The prognostic significance of RON and MET coexpression in patients with colorectal cancer. Dis Colon Rectum 5: 1268-1274, 2008.

24. Willett CG, Wang MH,Emanuel RL, et al: Macrophage-stimulating protein and its receptor in non-small cell lung tumors: induction of receptor tyrosine phosphorylation and cell migration. Am J Respir Cell Mol Biol 18: 489-496, 1998.

25. Maggiora P, Lorenzato A, Fracchioli S, et al: The RON and MET oncogenes are co-expressed in human ovarian carcinomas and cooperate in activating invasiveness. Exp Cell Res 288: 382-389, 2003.

26. Thomas RM, Toney K, Fenoglio-Preiser C, et al: The RON receptor tyrosine kinase mediates oncogenic phenotypes in pancreatic cancer cells and is increasingly expressed during pancreatic cancer progression. Cancer Res 67: 6075-6082, 2007.

27. Cheng HL, Liu HS, Lin YJ, et al: Co-expression of RON and MET is a prognostic indicator for patients with transitional-cell carcinoma of the bladder. Br J Cancer 92: 1906-1914, 2005.

28. Chen Q, Seol DW, Carr B and Zarnegar R: Co-expression and regulation of Met and Ron proto-oncogenes in human hepatocellular carcinoma tissues and cell lines. Hepatology 26: 59-66, 1997.

29. Leonis MA, Toney-Earley K, Degen SJ and Waltz SE: Deletion of the Ron receptor tyrosine kinase domain in mice provides protection from endotoxin-induced acute liver failure. Hepatology 36: 1053-1060, 2002 .

30. Stuart WD, Kulkarni RM, Gray JK, Vasiliauskas J, Leonis MA and Waltz SE: Ron receptor regulates Kupffer cell-dependent cytokine production and hepatocyte survival following endotoxin exposure in mice. Hepatology 53: 1618-1628, 2011.

31. Zimmermann $\mathrm{S}$ and Moelling K: Phosphorylation and regulation of Raf by Akt (protein kinase B). Science 286: 1741-1744, 1999.

32. Moelling K, Schad K, Bosse M,Zimmermann S and Schweneker M: Regulation of Raf-Akt Cross-talk. J Biol Chem 277: 31099-31106, 2002.

33. Mason EF and Rathmell JC: Cell metabolism: an essential link between cell growth and apoptosis. Biochim Biophys Acta 1813: 645-654, 2011 .

34. DeBerardinis RJ, Lum JJ, Hatzivassiliou G and Thompson CB: The biology of cancer: metabolic reprogramming fuels cell growth and proliferation. Cell Metab 7: 11-20, 2008.

35. Morgan DO: Principles of CDK regulation. Nature 374: 131-134, 1995.

36. Grana X and Reddy EP: Cell cycle control in mammalian cells: role of cyclins, cyclin dependent kinases (CDKs), growth suppressor genes and cyclin-dependent kinase inhibitors (CKIs). Oncogene 11: 211-219, 1995.

37. Song G, Ouyang G and Bao S: The activation of Akt/PKB signaling pathway and cell survival. J Cell Mol Med 9: 59-71, 2005.

38. Chappell WH, Steelman LS, Long JM, et al: Ras/Raf/MEK/ERK and PI3K/PTEN/Akt/mTOR inhibitors: rationale and importance to inhibiting these pathways in human health. Oncotarget 2: 135-164, 2011.

39. Chung E and Kondo M: Role of Ras/Raf/MEK/ERK signaling in physiological hematopoiesis and leukemia development. Immunol Res 49: 248-268, 2011. 